\title{
Ekbom Syndrome: the connection between Psychiatry and Dermatology - case report
}

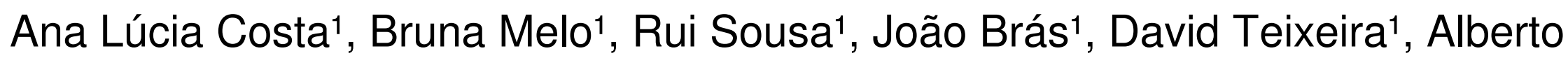
Marques $^{1}$, Carla Alves Pereira ${ }^{1}$, Ana Isabel Oliveira ${ }^{1}$, Jorge Humberto Silva ${ }^{1}$ 1. Department of Psychiatry and Mental Health, Centro Hospitalar Tondela-Viseu, EPE., Viseu, Portugal.

\section{BARKBRIIND AND AIMS}

Ekbom Syndrome (ES) or Delusional parasitoses (DP) is a rare psychiatric disorder that is characterised by the conviction of being infected with parasites or other living organisms, despite clear evidence of the contrary. It is commonly observed among patients over the age 50 and it is mainly described in presenile women. These patients experience visual and tactile hallucinations (they see and/or feel the bugs). In the DSM-5 it is classified as a delusional disorder, somatic type.

In primary DP, the delusion occurs independently of any medical condition; in secondary DP, the delusions arise in the setting of another major medical conditions, neurological condition or psychiatric disorder such as schizophrenia or affective disorders. Traditionally, it is treated with antipsychotic Pimozide, however, due to its side effects atypical antipsychotics could be considered as alternative option.

\section{DRAERTIVES}

To report a case of Ekbom Syndrome (ES) and an unsystematic review of the literature.

\section{MATERIAL AND MEHHDSS}

Case report. An unsystematic review was conducted consulting the search engine Pubmed® and the keywords: Ekbom Syndrome and Delusional Parasitosis.

\section{RESULISS}

0

76 years-old
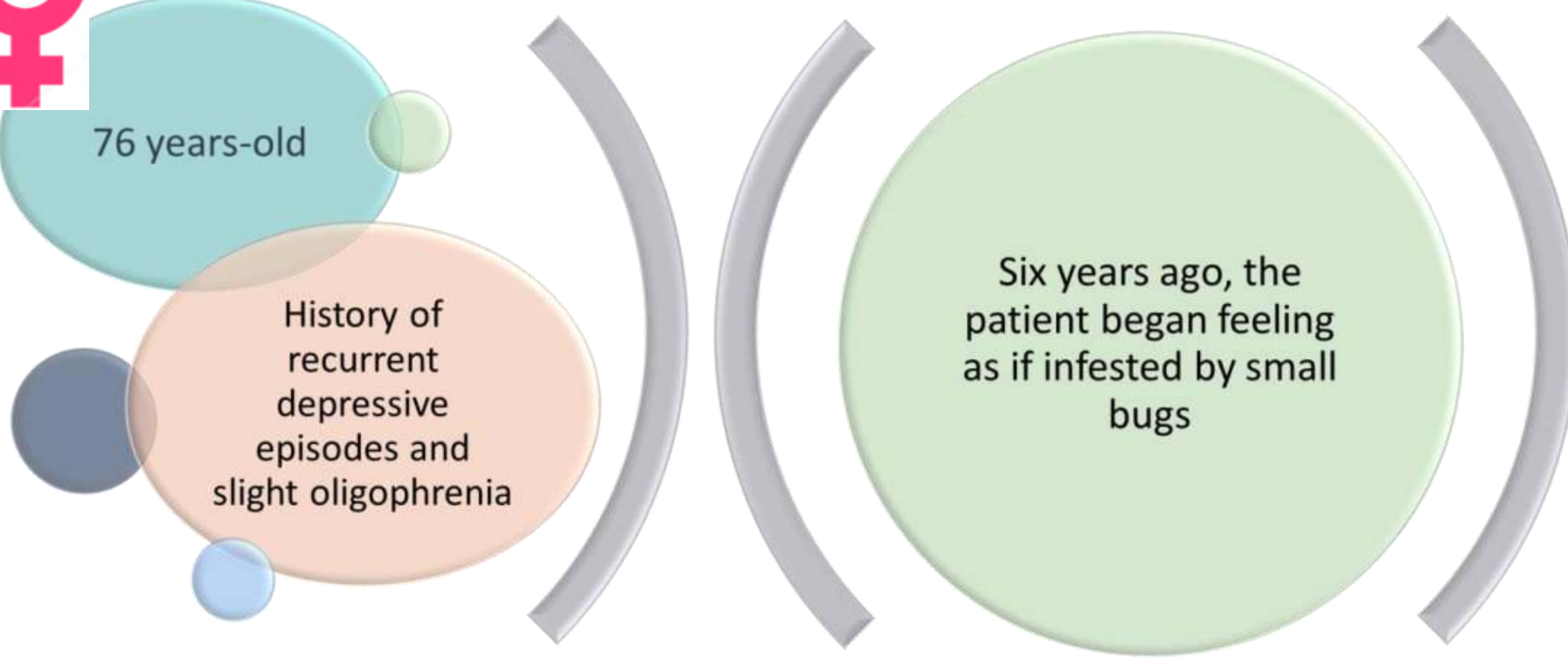

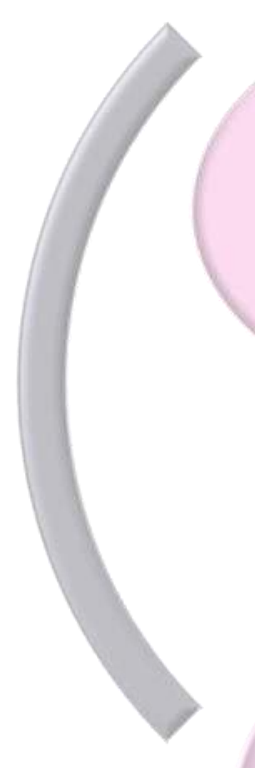

She referred that they were spread

throughout her body

and amassed under

her skin (head and genitals).

Increased anxiety, sleeplessness, elaborate delusions of being infested by bugs and visual/tactile hallucinations that bugs are crawling over her skin (formication). $x$

Risperidone 6mg/day without any improvement

Pimozide 2mg/day and then increased to $4 \mathrm{mg} /$ day + haldol 15mg/day

Although she continued to believe that bugs were still alive in her body, she was markedly less distressed.

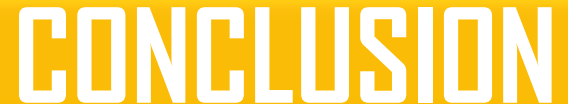

ES tends to be under-diagnosed and its diagnostic and treatment are difficult as patients with this disorder reject psychiatric diagnosis and they are convinced of having a dermatological problem.

Traditionally, DP is treated with antipsychotic Pimozide. However, it is associated with extrapyramidal side effects and also can cause prolongation of the QT interval, requiring periodic electrocardiographic monitoring so, usually it is the second choice. Due to these side effects, use of atypical antipsychotics, such as Risperidone, should be considered as first choice. In addition to drug therapy, establishing a therapeutic relationship with the patient is crucial.

Awareness about this syndrome is important to all physicians to get earlier detection and rationalize the treatment. 